\title{
Tinea Capitis: A Practical Approach
}

\section{Juarez Duran ER, Asz Sigall D, Vega DC and Arenas R* \\ Mycology Section, Hospital General Dr. Manuel Gea Gonzalez, Mexico}

*Corresponding author: Roberto Arenas, Mycology Section, Hospital General Dr. Manuel Gea Gonzalez, Tlalpan 4800 CD Mex, Mexico, Email: rarenas98@hotmail.com

\section{Mini Review}

Volume 2 Issue 2

Received Date: December 17, 2019

Published Date: December 31, 2019

DOI: $10.23880 /$ oajmms-16000113

\section{Abstract}

Tinea capitis is a common superficial fungal infection of the scalp in children. It is caused by dermatophytes mainly Microsporum canis and Trichophyton tonsurans and can be acquired from the environment or infected living beings. Clinical manifestations go from mild scaling with little hair loss to large inflammatory and alopecic pustular plaques. Trichoscopic patterns such as comma hairs, corkscrew or code-like hairs can suggest and support the diagnosis as well as clinical follow up. Treatment must be chosen due to causal agent for a successful outcome.

Keywords: Trichophyton spp; Microsporum spp; Dermatophytes; Trichophyton Tonsurans

\section{Introduction}

Tinea capitis is a superficial fungal infection of the scalp, hair shaft, eyebrows and eyelashes, also referred as ringworm and it remains a major public health concern in urban areas. It is caused by dermatophytes of Trichophyton and Microsporum genera [1,2].

It remains the most common superficial fungal infection in children. The causal agent's distribution has diverse geographical variations depending on several factors such as socio-economic level, population, migration among others.

Infection can be acquired from the environment, wild animals, pets or people with this dermatophytes infection. Also, there are specific conditions that favor the growth of the fungus such as humidity, heath, extended use of corticosteroids and lack of hygiene [3].

Clinical manifestations are varied from mild scaling with little hair loss to large inflammatory and pustular plaques with extensive alopecia. A simple non-invasive technique for diagnosis and follow-up is trichoscopy. Specific aspects of the hair have been described like: comma hair, corkscrew hair, bar code-like hair and zigzag hair $[4,5]$.

Diagnosis is achieved by focused clinical history, clinical examination, mycological and trichoscopical study. Treatment will depend on causal agent, age and weight.

Our goal is to analyze current data within the last years about this infection.

\section{Epidemiology}

Principal changes related to epidemiology of tinea capitis in latest years has been the rise of M. canis as the dominant fungal agent in some parts of Europe and the spread of T. tonsurans in urban communities in USA, Western Europe, UK and France [6,7].

T. tonsurans has spread to both South America and West Africa [8,9]. In Far Eastern countries has mainly been associated with infection in older children and wrestling in adults [10]. Trends in tinea capitis and migration, has also increase infections due to Trichophyton violaceum and Microsporum audouinii in countries that were previously not common $[11,12]$. 


\section{Open Access Journal of Mycology \& Mycological Sciences}

However, the rise in tinea capitis due to T. tonsurans in any country has presented a challenge to both treatment and community infection control.

\section{Clinical Features}

Tinea capitis is caused by many species of dermatophytes which infect hair follicles and contiguous skin. It affects children from 2to12 years old, but predominantly between 3-7 and it is more commonly observed in a low socioeconomic population $[2,13]$.

Even though this fungal infection it's considered an "only child's" disease, a growing number of tinea capitis cases have been reported in the past years among adults, particularly menopausal, elderly women and immunocompromised patients [13].

The clinical appearance of tinea capitis will in part depend on the type of invasion affecting our patient [14].

- Ectothrix Form: The hair shaft is infected at midfollicle level and hyphae grow towards hair bulb. Fluorescence under Wood's light its characteristically present in infections caused by Microsporum species. There is hair loss with hair shafts breaking 2-3 mm above the scalp level.

- Endothermic Form: Almost all hairs break at scalp level, leaving swollen hair stubs inside the follicles appearing "black dots". Trichophyton species does not fluorescence under Wood's light [15].

Another clinical feature will depend on level of host resistance and inflammatory host response; it will vary from undetectable with a few broken hairs, little scaling and inflammation to a severe, painful, boggy mass.

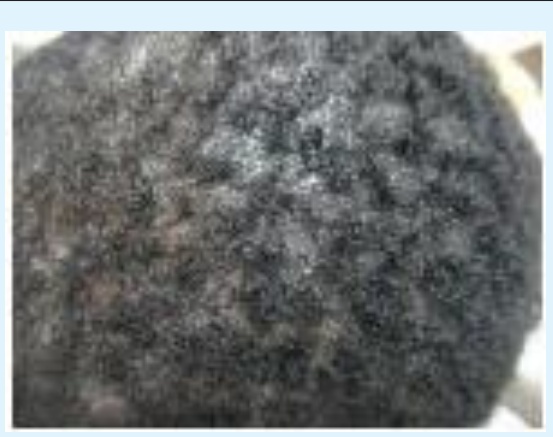

Figure 1: Trichophytic tinea due to T. tonsurans.

Arenas R, et al. Tinea Capitis: A Practical Approach. J Mycol Mycological Sci 2019, 2(2): 000113.

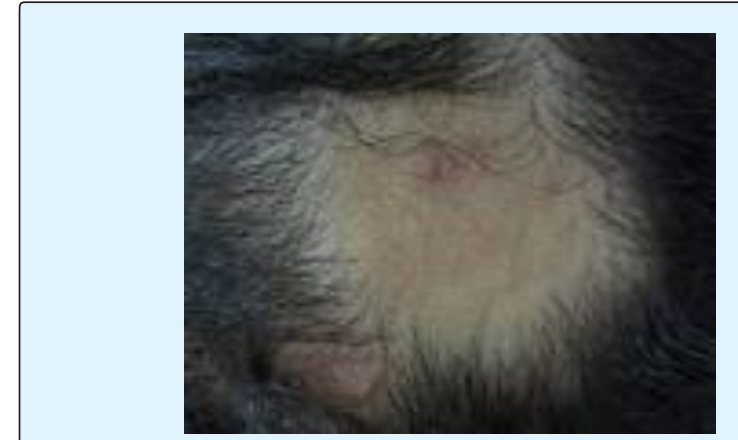

Figure 2: Microsporic tinea due to $M$. canis.

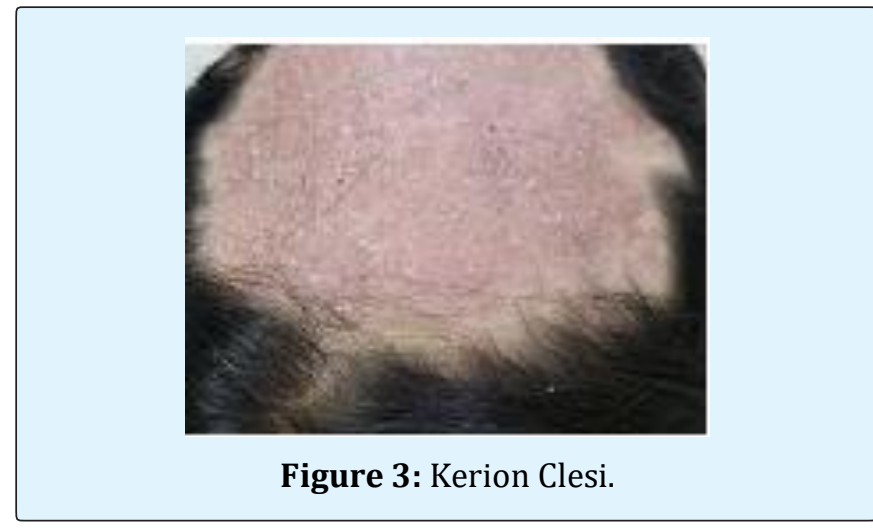

Tinea capitis can be classified in two types: Dry variety (conformed by "microsporic tinea" and "trichophytic tinea"), mainly cause by Mycrosporum canis and Trichophyton tonsurans. Inflammatory variety also called Kerion Celsi, mainly caused by T. mentagrophytes, M. canis and rarely T. verrucosum. In all types partial hair loss is present [15] (Figures 1-3).

\section{Dry, Non-Inflammatory Variety}

- Microsporum specie: Lesions can be alopecia patches mostly circular, broken hairs at same height and characteristic fine scaling but little inflammation

- Trichophyton specie: Lesions can be diffuse alopecia with little and irregular plaques interspersed with healthy hairs.

\section{Trichocospic Features}

Trichoscopy allows in vivo visualization infected hair patterns and epidermal portion of hair follicles, which cannot be seen by the naked eye $[13,16]$.

Typical trichoscopic findings can support the diagnosis but mycological examination remains the gold standard for confirmation of tinea capitis $[17,18]$. 
One of the main dermoscopic markers of tinea capitis is comma hairs, which appear as short C-shaped hairs due to disintegrated, cracked, and bent hairs caused by multiple hyphae within the hair shaft. Corkscrew hairs were proposed as new trichoscopy finding of tinea capitis in patients of African descent due the shape of the broken hair shaft. Nevertheless, Hughes et al. declared that comma hair and corkscrew are the main trichoscopic findings; they pointed out that corkscrew hair is a variant of comma hair in African patients and is a marker for endothrix tinea capitis [17-19]. But in our experience both patterns can be found in Caucasians, blacks and mestizo patients (Figures $4 \& 5$ ).

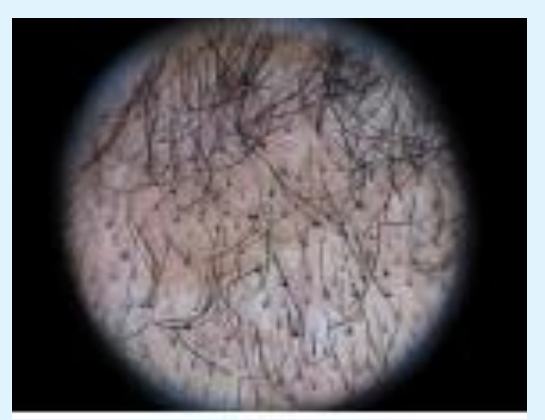

Figure 4: Dermoscopic findings, comma and corkscrew hairs.

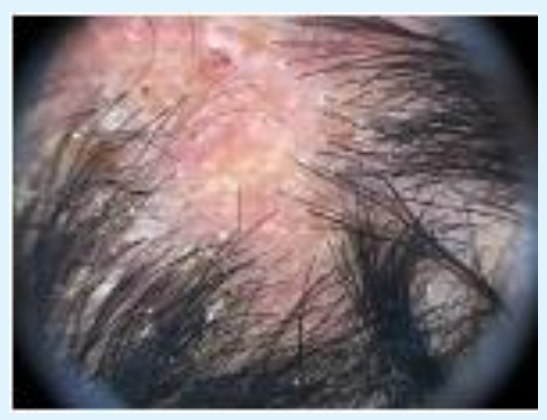

Figure 5: Kerion Celsi, zig zag hairs.

The recently described bar code-like hairs also called Morse code hair is a specific dermoscopic patterns of fungal infection of the scalp. The features of the bar codelike hair are horizontal white bands which can be better observed by polarized dermoscopic. Under ultraviolet dermoscopy bright-green fluorescence in hair shaft is present and bright-white in accumulated a scale, that disappears at the bands of the bar code-like hairs [20].
Campos $S$ et al performed a non-randomized prospective clinical study with fifty patients under 13 years of age and the most frequent dermoscopic findings were perifollicular scaling in $94 \%$, diffuse scaling $90 \%$, short broken hairs $74 \%$, black dots $34 \%$, corkscrew hairs $30 \%$, comma hairs $20 \%$, pustules $18 \%$ and zigzag hairs $12 \%$ [21]. After treatment a progressive disappearance of hair alterations it's observed and at follow-up all patients had a negative mycological examination and dystrophic hairs were not observed [21].

Richarz N, et al. [22] proposed trichoscopy as a fast, non-invasive method for monitoring treatment; they reported the resolution of corkscrew hair after an antifungal therapy. It predicts a clinical cure accurately, allowing us to decide to stop antifungal oral therapy.

\section{Treatment}

Tinea capitis treatment should be started once the clinical diagnosis and the direct examination with $\mathrm{KOH}-$ Chlorazol black are confirmative. It is not recommended to wait for the fungal culture to grow because delaying treatment increases the risk for disease progression, permanent hair loss, and transmission $[3,23]$.

There is very low or none clinical improvement with antifungals such as voriconazole or posaconazole. Tinea capitis require clinical follow-up to determine if oral antifungal treatment should be discontinued, changed or if additional treatment is needed [23,24].

Topical therapy alone is not recommended, they have an inadequate penetration of the hair follicles and may miss areas of subclinical infection. Povidone-iodine, ketoconazole $2 \%$, selenium sulfide $1-2.5 \%$ and ciclopirox shampoo have shown some efficacy to reduce the risk of spreading, transmission to other children and decreasing the risk for reinfection $[25,26]$.

As a general rule in tinea capitis, terbinafine is more effective against Trichophyton spp ( $T$. tonsurans, $T$. violaceum, T. soudanense), as opposed to griseofulvin and itraconazole which are more effective against Microsporum spp (M. canis, M. audouinii) [3].

Griseofulvin and terbinafine are the two drugs approved by the US Food and Drug Administration (FDA) and the mainly used by the same reason. Ketoconazole should not be used for tinea capitis, because of the risk of severe liver injury and adrenal insufficiency $[3,19,23,24,27]$. 


\section{Open Access Journal of Mycology \& Mycological Sciences}

\section{First Line Treatment}

Optimal treatment must be chosen according to the etiological dermatophyte involved $[3,23,24,27]$ (Table 1 ).

\begin{tabular}{|c|c|}
\hline Trichophyton spp. & Terbinafine \\
\hline Trichophyton tonsurans & \\
\hline Trichophyton violaceum / soudanense & \\
\hline Microsporum spp. & $\begin{array}{c}\text { Griseofulvine or } \\
\text { Itraconazole }\end{array}$ \\
\hline Trichophyton spp. & Terbinafine \\
\hline Trichophyton tonsurans & \\
\hline
\end{tabular}

Table 1: Treatment in tinea capitis depending causal agent.

Griseofulvin: It is a fungi static drug with micronized, ultra-micronized and suspension forms, not always available in some countries [28]. The recommended dose in children is 15 to $20 \mathrm{mg} / \mathrm{kg}$ per day in single or divided doses for 6 to 8 weeks. In resistant cases doses up to $25 \mathrm{mg} / \mathrm{kg}$ can be necessary. Griseofulvin effectiveness is low against Trichophyton spp. [29]. A meta-analysis comparing griseofulvin and terbinafine has shown that griseofulvin should be the first-line treatment for children in whom the etiological agent is unclear [30].

Griseofulvin is contraindicated in lupus erythematosus, porphyria, liver disease and pregnancy $[29,31]$.

Terbinafine: Terbinafine is an alternative first-line treatment and considered the optimal choice, due to shorter courses, cost-efficiency, and compliance [29]. A meta-analysis showed that the effectiveness of 2to 4 week's treatment with terbinafine is similar to the 6to8 week's treatment with griseofulvin for T. tonsurans [3235]. A new granule formulation available in $125-\mathrm{mg}$ or 187.5-mg packets to be sprinkled on food has been licensed in the USA for children over four years of age $[23,30,36]$.

\section{Second Line Treatment}

Itraconazole: It has both fungicidal and fungistatic activity. Doses of 50 to 100 daily $\mathrm{mg}$ for four weeks have comparable efficacy with griseofulvin or terbinafine $[23,31]$.

Pulse intermittent dosing regimens are effective. This is recommended at $5 \mathrm{mg} / \mathrm{kg}$ per day for one week each month during two to three months. In the U.K. it is not recommended for the treatment of tinea capitis in children aged 12 years and under [23,39]. Liver function tests are indicated in patients treated with azolic derivates for over a month or before starting treatment in patients with pre-existing hepatic dysfunction $[3,39,40]$.

Fluconazole: It is considered an alternative to terbinafine. It can be administered as a pulse therapy of $6 \mathrm{mg} / \mathrm{kg}$ once a week for 6 to 12 weeks. Serious side effects including hepatoxicity, severe drug hypersensitivity reactions (Stevens-Johnson syndrome), anaphylaxis, torsade de pointes, and prolongation of the QT interval, are rare [23,41-43].

Infants under the Age of 1 Year: Tinea capitis is uncommon in infants under the age of one year and there is no a consensus about treatment although Fluconazole, griseofulvin, and terbinafine have shown efficacy in this population $[44,45]$.

Treatment in Adults: Drug selection and treatment duration criteria in adult tinea capitis are similar to the ones used for children; however, optimal regimens still unclear. The recommended doses of antifungals in adults are: Terbinafine $250 \mathrm{mg}$ per day, Itraconazole $5 \mathrm{mg} / \mathrm{kg}$ per day (maximum $400 \mathrm{mg} /$ day), fluconazole $6 \mathrm{mg} / \mathrm{kg}$ per day, and ultra-micronized griseofulvin 10 to $15 \mathrm{mg} / \mathrm{kg}$ per day (maximum 750mg/day) [23,41-43].

Kerion Celsi: It is recommended to combine antifungal therapy with oral prednisone in order to prevent scaring alopecia in $0.5 \mathrm{mg} / \mathrm{kg} /$ day for two weeks. Wet compresses are useful to remove exudates and crusts when bacterial infection its suspected. In small cases, topical or systemic antibiotics can be prescribed $[23,44,45]$.

\section{Comments}

Tinea capitis is a fungal infection caused by dermatophytes of the genera Microsporum spp and Trichophyton spp. The hair shaft is infected as Ectothrix form in the first one and Endothrix form in the second one. Clinical manifestations can be classified in two types: the dry variety (microsporic and trichophytic), and the inflammatory form (kerion Celsi). Trichoscopic findings as comma hairs, corkscrew and code-like hairs can support clinical diagnosis. Treatment must be chosen according with causal agents, terbinafine is more effective against Trichophyton spp and griseofulvin and itraconazole are more effective against Microsporum spp.

\section{References}

1. Wolff K, Lowell AG, Katz SI, Gilchrest BA, Paller AS (2008) Fitzpatrickss Dermatology in general medicine. Dermatologic Surgery 34(8): 1081. 


\section{Open Access Journal of Mycology \& Mycological Sciences}

2. Guzman RA (2015) Dermatology. Atlas, diagnosis and treatment, $6^{\text {th }}$ (Edn.), Access Medicine Mexico, pp: 505-518.

3. Fuller LC, Barton RC, Mohd Mustapa MF, Proudfoot LE, Punjabi SP, et al. (2014) British association of Dermatologists' guidelines for the management of tinea capitis 2014. Br J Dermatol 171(3): 454-463.

4. Gupta AK, Mays RR, Verseeg SG, Piraccini BM, Shear $\mathrm{NH}$, et al. (2018) Tinea capitis in children: a systematic review of management. J Eur Acad Dermatol Venereol 32(12): 2264-2274.

5. Bourezane Y (2017) Analysis of trichoscopic signs observed in 24 patients presenting tinea capitis: Hypotheses based on physiopathology and proposed new classification. Ann Dermatol Venereol 144(8-9): 490-496.

6. Fuller LC (2009) Changing face of tinea capitis in Europe. Curr Opin Infect Dis 22(2): 115-118.

7. Alshawa K, Lacroix C, Benderdouche M, Mingui A, Derouin F, et al. (2012) Increasing incidence of Trichophyton tonsurans in Paris, France: a 15-year retrospective study. Br J Dermatol 166(5): 1149. 1150.

8. Sidrim JJ, Rocha MF, Leite JJ, Maranhao FC, Lima RA, et al. (2013) Trichophyton tonsurans strains from Brazil: phenotypic eterogeneity, genetic homology, and detection of virulence genes. Can J Microbiol 59(11): 754-760.

9. Hogewoning AA, Adegnika AA, Bouwes Bavinck JN, Yazdanbakhsh M, Kremsner PG, et al. (2011) Prevalence and causative fungal species of tinea capitis among schoolchildren in Gabon. Mycoses 54(5): 354-359.

10. Mapelli ET, Cerri A, Bombonato C, Menni S (2013) Tinea capitis in the paediatric population in Milan, Italy: the emergence of Trichophyton violaceum. Mycopathologia 176(3-4): 243-246.

11. Kieliger S, Glatz M, Cozzio A, Bosshard PP (2015) Tinea capitis and tinea faciei in the Zurich area-an 8year survey of trends in the epidemiology and treatment patterns. J Eur Acad Dermatol Venereol 29(8): 1524-1529.

12. Sei Y (2015) 2011 Epidemiological survey of Dermatomycoses in Japan. Med Mycol J 56(4): 129135.
13. Elghblawi E (2017) Tinea capitis in children and trichoscopic criteria. Int J Trihcology 9(2): 47-49.

14. Kaushik N, Pujalte GGA, Reese ST (2015) Superficial fungal infections. Prim Care Clinics in office practice 42(4): 501-516.

15. Hay RJ (2017) Tinea capitis current status. Mycopathologia 182(1-2): 87-93.

16. Amer M, Helmy A, Amer A (2017) Trichoscopy as a useful method to differentiate tinea capitis from alopecia areata in children at Zagazig University Hospitals. Int J Dermatol 56(1): 116-120.

17. Elghblawi E (2016) Idiosyncratic findings in Trichoscopy of tinea Capitis: comma, zigzag hairs, corkscrew, and Morse code-like hair. Int J Trichology 8(4): 180-183.

18. Hughes R, Chiaverini C, Bahadoran P, Lacour JP (2011) Corkscrew hair: A new dermoscopic sing for diagnosis of tinea capitis in black children. Arch Dermatol 147(3): 355-356.

19. Tosti A (2015) Dermoscopy of the hair and nails. $2^{\text {nd }}$ (Edn.), Taylor \& Francis group USA, pp: 220.

20. Tang J, Ran X, Ran Y (2017) Ultraviolet dermoscopy for the diagnosis of tinea capitis. J Am Acad Dermatol 76(2S1): 28-30.

21. Campos S, Brasileiro A, Galhardas C, Apetato M, Cabete J, et al. (2017) Follow-up of tinea capitis with trichoscopy: a prospective clinical study. J Eur Acad Dermatol Venerol 31(11): 478-480.

22. Richarz N, Barboza L, Monsonis M, Gonzalez Ensenat MA, Vicente A (2018) Trichoscopy helps to predict the time point of clinical cure of tinea capitis. Australas J Dermatol 59(4): 298-299.

23. Treat JR (2019) Tinea capitis. In: Rosen T, Levy ML, (Eds.), UpTo Date.

24. Lorch Dauk KC, Comrov E, Blumer JL, O’Riordan MA, Furman LM (2010) Tinea capitis: predictive value of symptoms and time to cure with griseofulvin treatment. Clin Pediatr 49(3): 280-286.

25. Greer DL (2000) Successful treatment of tinea capitis with 2\% ketoconazole shampoo. Int J Dermatol 39(4): 302-304.

26. Chen C, Koch LH, Dice JE, Dempsey KK, Moskowitz AB, et al. (2010) A randomized, double-blind study comparing the efficacy of selenium sulfide shampoo 


\section{Open Access Journal of Mycology \& Mycological Sciences}

$1 \%$ and ciclopirox shampoo $1 \%$ as adjunctive treatments for tinea capitis in children. Pediatr Dermatol 27(5): 459-462.

27. Fuller LC, Smith $\mathrm{CH}$, Cerio R, Marsden RA, Midgley G, et al. (2001) A randomized comparison of 4 weeks of terbinafine vs. 8 weeks of griseofulvin for the treatment of tinea capitis. Br J Dermatol 144(2): 321327.

28. Gupta AK, Cooper EA, Bowen JE (2008) Meta-analysis: griseofulvin efficacy in the treatment of tinea capitis. J Drugs Dermatol 7(4): 369-372.

29. Kakourou T, Uksal U (2010) Guidelines for the management of tinea capitis in children. Pediatr Dermatol 27(3): 226-228.

30. Gupta AK, Main DC (2013) Meta-analysis of randomized, controlled trials comparing particular doses of griseofulvin and terbinafine for the treatment of tinea capitis. Pediatr Dermatol 30(1): 16.

31. Gonzalez U, Seaton T, Bergus G, Jacobson J, Monzon MC (2007) Systemic antifungal therapy for tinea capitis in children. Cochrane Database Syst Rev 5: CD004685.

32. Friedlander SF, Aly R, Krafchik B, Blumer J, Honig P, et al. (2002) Terbinafine in the treatment of Trichophyton tinea capitis: a randomized, doubleblind, parallel-group, duration-finding study. Pediatrics 109(4): 602-607.

33. Devliotou Panagiotidou D, Koussidou Eremondi TH (2004) Efficacy and tolerability of 8 weeks' treatment with terbinafine in children with tinea capitis caused by Microsporum canis: a comparison of three doses. J Eur Acad Dermatol Venereol 18(2): 155-159.

34. Arabatzis M, Kyprianou M, Velegraki A, Makri A, Voyatzi A (2010) Microsporum canis antifungal susceptibilities: concerns regarding their clinical predictability. Int J Antimicrob Agents 36(4): 385386.

35. Hanselmayer GG, Seebacher C (2011) Treatment of tinea capitis-a critical appraisal. J Dtsch Dermatol Ges 9(2): 109-114.

36. Elewski BE, Caceres HW, DeLeon L, El Shimy S, Hunter JA, et al. (2008) Terbinafine hydrochloride oral granules versus oral griseofulvin suspension in children with tinea capitis: results of two randomized, investigator-blinded, multicenter, international, controlled trials. J Am Acad Dermatol 59(1): 41-54.

37. Hanselmayer GG, Smolle J, Gupta A (2004) Itraconazole in the treatment of tinea capitis caused by Microsporum canis: experience in a large cohort. Pediatr Dermatol 21(4): 499-502.

38. Mathioudaki KE, Panagiotidou DD, Rallis E, Athanassopoulou V, Eremondi KT, et al. (2005) Is itraconazole the treatment of choice in Microsporum canis tinea capitis?. Drugs Exp Clin Res 31: 11-15.

39. Gupta AK, Cooper EA (2008) Update in antifungal therapy of dermatophytosis. Mycopathologia 166(56): 353-367.

40. Grover C, Arora P, Manchanda V (2012) Comparative evaluation of griseofulvin, terbinafine and fluconazole in the treatment of tinea capitis. Int J Dermatol 51(4): 455-458.

41. Gupta AK, Dlova N, Taborda P, Morar N, Taborda V, et al. (2000) Once weekly fluconazole is effective in children in the treatment of tinea capitis: a prospective, multicentre study. Br J Dermatol 142(5): 965-968.

42. Gilaberte Y, Rezusta A, Gil J, Saenz Santamaria MC, Coscojuela C, et al. (2004) Tinea capitis in infants in their first year of life. Br J Dermatol 151(4): 886-890.

43. Zaraa I, Hawilo A, Trojjet S, El Euch D, Mokni M, et al. (2012) Letter: Tinea capitis in infants in their first 2 years of life: A 12-year study and a review of the literature. Dermatol Online J 18(7): 16.

44. Keipert JA (1984) Beneficial effect of corticosteroid therapy in microsporum canis kerion. Australas J Dermatol 25(3): 127-130.

45. Proudfoot LE, Higgins EM, Morris Jones R (2011) A retrospective study of the management of pediatric kerion in Trichophyton tonsurans infection. Pediatr Dermatol 28(6): 655-657. 